\title{
Rivastigmine in moderately severe-to-severe Alzheimer's disease: Severe Impairment Battery factor analysis
}

\author{
Steven Ferris ${ }^{1 *}$, Stella Karantzoulis ${ }^{1}$, Monique Somogyi ${ }^{2}$ and Xiangyi Meng ${ }^{2}$
}

\begin{abstract}
Introduction: The Severe Impairment Battery (SIB) is validated for assessing cognition in patients with severe dementia. The current analysis aimed to further investigate the cognitive efficacy of rivastigmine capsules, as assessed by SIB factor scores, in patients with moderately severe-to-severe Alzheimer's disease (AD).
\end{abstract}

Methods: This was a retrospective analysis of a 26-week, multicenter, randomized, double-blind, placebo-controlled study of oral rivastigmine conducted in Spain. Previously reported outcome measures included the full SIB. Current analyses examined calculated scores and effect sizes for the change from baseline at Week 26 on: newly defined SIB subscales (derived by a factor analysis of the 40 SIB items, using the PROC FACTOR function (SAS)); previously defined memory, language and praxis subscales (derived by previous analysis of the nine SIB domains); and the individual SIB items. Treatment differences were assessed.

Results: SIB data were provided by 104 rivastigmine-treated patients and 106 patients receiving placebo (Intent-To-Treat Last Observation Carried Forward population). Significantly less decline was observed on the previously defined memory and language subscales, and the newly defined working memory/memory subscale in rivastigmine-treated patients (all $P<0.05$ versus placebo). Calculation of effect sizes demonstrated numerically greater efficacy of rivastigmine versus placebo on each of the subscales, and a broad range of SIB items; greatest effect sizes were observed on SIB items assessing the current month (effect size $=0.30$ ) and digit span series (effect size $=0.33$ ).

Conclusions: These data suggest the observed efficacy of rivastigmine in moderately severe-to-severe AD is likely a cumulative effect across a range of tasks. Rivastigmine demonstrates broad cognitive efficacy in this patient population.

\section{Introduction}

The cholinesterase inhibitor rivastigmine has demonstrated efficacy on activities of daily living (ADL), cognition and behavior in clinical trials of patients with mild-to-moderately severe Alzheimer's disease (AD) [1-4]. Currently, oral (6 to $12 \mathrm{mg} /$ day) and transdermal rivastigmine (4.6 mg/24 h, $9.5 \mathrm{mg} / 24 \mathrm{~h}$ and $13.3 \mathrm{mg} / 24 \mathrm{~h}$ ) are approved in the USA for the symptomatic treatment of mild-to-moderate $\mathrm{AD}[5,6]$. A previously reported pooled analysis of data from clinical trials investigating

\footnotetext{
* Correspondence: steven.ferris@nyumc.org

'Alzheimer's Disease Center, Comprehensive Center on Brain Aging, NYU Langone Medical Center, Room 506, 145 East 32nd Street, New York, NY 10016, USA

Full list of author information is available at the end of the article
}

the efficacy of rivastigmine in patients with mildto-moderately severe $\mathrm{AD}$ suggested that rivastigmine may also benefit patients at more advanced stages of the disease [7]. Indeed, the rivastigmine transdermal patch has recently been approved in the USA for the symptomatic treatment of severe AD [5]. This approval was based on the findings of the ACTivities of daily living and cognitION (ACTION) study, a large, 24-week, multicenter, randomized, active-controlled trial assessing the comparative efficacy and safety of $13.3 \mathrm{mg} / 24 \mathrm{~h}$ versus $4.6 \mathrm{mg} / 24 \mathrm{~h}$ rivastigmine transdermal patch in patients with severe $\mathrm{AD}[8]$.

The efficacy and safety of oral rivastigmine in Spanish patients with moderately severe-to-severe AD (MiniMental State Examination (MMSE) score of 5 to 12) were 
previously investigated in a 26-week, multicenter, randomized, double-blind, placebo-controlled trial [9]. In this study, rivastigmine treatment was associated with significantly less decline on the Severe Impairment Battery (SIB) and significant improvements on the MMSE and Alzheimer's Disease Cooperative StudyClinical Global Impression of Change (ADCS-CGIC) scale, compared with placebo [9]. A trend towards improvement, or reduced decline, was also seen in rivastigmine-treated patients compared with placebo on the 10-item Neuropsychiatric Inventory (NPI-10), the 4-item NPI (NPI-4), the Global Deterioration Scale (GDS) and the Alzheimer's Disease Cooperative StudyActivities of Daily Living (ADCS-ADL) scale [9]. Of the assessment scales used in this study, the SIB was specifically designed and validated for use in patients with severe dementia, since they may have difficulty completing more challenging standard neuropsychological assessments, [10-12] such as the Alzheimer's Disease Assessment Scale-cognitive subscale (ADAS-cog).

The SIB is a performance-based measure that assesses nine symptom domains: attention, language, orientation, memory, praxis, visuospatial perception, construction, social skills and orientating head to name [10-12]. Several variations of this test have been developed, with a range of possible total scores [10-12]. One such version of the SIB assesses patients on 40 items (questions) to give a total score ranging from 0 to 100 , where a lower score is indicative of more severe impairment [11].

Previous analyses have grouped the nine domains of the SIB into three higher-order subscales: memory, language and praxis (Table 1) [13]. However, the specific cognitive functions comprising these higher-order subscales are broad; for example, the language domain contains items that assess more than just language (for example, naming the months of the year, which could also be considered a memory assessment). Wider domain coverage is also true for the memory and praxis

Table 1 Previous organization of the SIB domains into higher-order subscales [13]

\begin{tabular}{ll}
\hline Higher-order subscale & SIB domain \\
\hline Memory & Memory \\
& Attention \\
& Orientation \\
& Orientating head to name \\
& Language \\
Language & Social skills \\
& Praxis \\
Praxis & Construction \\
& Visuospatial perception \\
\hline
\end{tabular}

SIB, Severe Impairment Battery. subscales. Redefining the subscales of the SIB using a new factor analysis of the 40 individual SIB items, as opposed to the broad SIB domains, as well as examining treatment effects on individual items, may facilitate further investigation of the efficacy of rivastigmine on more specific aspects of cognition in patients with severe $\mathrm{AD}$.

The aim of the current post-hoc analysis was to further investigate the efficacy of oral rivastigmine on cognition in severe $\mathrm{AD}$, using $\mathrm{SIB}$ item data from the previously reported clinical trial of oral rivastigmine in moderately severe-to-severe AD [9]. More specifically, the subscales of the SIB were redefined by performing a factor analysis of the individual items, as opposed to starting with the previously defined symptom domains. We investigated the pattern of efficacy of rivastigmine 1) on these newly defined subscales; 2) on the memory, language and praxis higher-order subscales of the SIB derived by a previous analysis [13]; and 3) for each of the individual SIB items.

\section{Methods}

This was a post-hoc analysis of a 26-week, multicenter, randomized, double-blind, placebo-controlled, parallelgroup study conducted in Spain that evaluated oral rivastigmine treatment in patients with moderately severe-to-severe AD [9]. The full details of the design and conduct of this study have been published previously [9]. Briefly, patients were male or female, aged 55 years or older, with probable AD (Diagnostic and Statistical Manual of Mental Disorders, $4^{\text {th }}$ edition and the National Institute of Neurological and Communicative Disorders and Stroke-Alzheimer's Disease and Related Disorders Association criteria) of moderately severe-to-severe intensity (MMSE score 5 to 12, GDS stage 5 to 6) [9]. Patients randomized to rivastigmine were up-titrated to the target dose of $6 \mathrm{mg}$ rivastigmine capsules twice-daily (bid) in four-week steps (using $1.5 \mathrm{mg}$ bid, $3 \mathrm{mg}$ bid and $4.5 \mathrm{mg}$ bid as intermediate doses) [9]. This titration schedule was modified in the event of any tolerability issues. Outcome measures included the change from baseline at Week 26 on the SIB [9]. This study received ethical committee approval and was conducted according to the ethical principles of the Declaration of Helsinki, as revised in 2000 [9].

Baseline SIB data from this study were used to perform a new factor analysis to establish a 'best fit' for each of the 40 individual SIB items into possible new subscales determined by the factor analysis. For this new factor analysis, the PROC FACTOR function in SAS was used. Initial common factor extraction was performed using the principal component method. Estimates of loadings were obtained using varimax rotation. Items 
were allocated to factors based on loadings and highest factor scores.

The least-squares (LS) mean change from baseline at Week 26 on the previously published (memory, language and praxis) [13] and newly defined subscales of the SIB were calculated for the Intent-To-Treat Last Observation Carried Forward (ITT-LOCF) population. Additional analyses were also performed based on the Observed Case (OC) population. For the ITT-LOCF approach, the statistical significance of treatment differences was assessed using an analysis of covariance (ANCOVA) model, with treatment and center as factors, and baseline as a covariate. For the ITT-OC approach, the statistical significance of treatment differences was assessed using a repeated measure ANCOVA model, with treatment, center, time-point and treatment-by-time point as factors, and baseline as a covariate. $P$-values were calculated from LS means.

Effect sizes (Cohen's d) were calculated, based on mean values, to compare the change from baseline at Week 26 on the newly defined SIB subscales, the previously defined SIB subscales and each of the individual items of the SIB in patients randomized to receive rivastigmine or placebo.

\section{Results}

\section{Patients}

In the 26-week, multicenter, randomized, double-blind, placebo-controlled, parallel-group trial on which this analysis is based, 218 patients were randomized (1:1) to receive rivastigmine or placebo [9]. Five rivastigminetreated patients and three patients in the placebo group did not provide a post-treatment SIB assessment and were excluded from the analyses. Therefore, the ITTLOCF population comprised 210 patients (104 in the rivastigmine group, and 106 in the placebo group) [9]. The ITT-OC population at Week 26 comprised 194 patients (96 in the rivastigmine group and 98 in the placebo group). At Week 26, the mean (standard deviation (SD)) dose of rivastigmine received by the rivastigmine group was 9.8 (2.8) $\mathrm{mg} /$ day [9].

At baseline, both treatment groups were similar in terms of their baseline demographics and patient characteristics. The mean age of the rivastigmine-treated group and the placebo group was 78.0 (range 60 to 89) and 77.2 (range 57 to 92 ) years, respectively. Seventy-eight percent of the rivastigmine-treated patients and $76 \%$ of the placebo group were female. The mean (SD) MMSE score at baseline was $9.0(2.2)$ in the rivastigmine group and 8.7 (2.2) in the placebo group, indicating moderately severe-to-severe impairment [9].

\section{Factor analyses}

The new factor analysis performed on the SIB at baseline allocated each of the 40 individual items to one of five factors. Allocation was based on which factor was associated with the highest factor score for each individual item (Table 2). The individual items allocated to each factor, and their corresponding SIB domain, are summarized in Table 3. Based on the individual items comprising these factors and their clinical relevance, we named Factor 1, visual; Factor 2, language; Factor 3, working memory/memory; Factor 4, praxis and social skills; and Factor 5, naming.

\section{Efficacy of rivastigmine on the previously defined memory,} language and praxis higher-order subscales of the SIB

Using the three subscales derived from a previous analysis [13], significantly reduced decline was seen on the memory (least-squares mean (standard error) change from baseline at Week 26: rivastigmine, -0.40 (0.46); placebo, $-1.92(0.47) ; P=0.010)$ and language (rivastigmine, -0.48 (0.76); placebo, -2.40 (0.77); $P=0.045)$ higherorder subscales of the SIB. A trend towards reduced decline, narrowly missing significance, was also observed on the praxis higher-order subscale of the SIB in rivastigmine-treated patients compared with placebo (rivastigmine, -0.33 (0.45); placebo, $-1.43(0.46) ; P=0.051$ ). Calculated effect sizes showed numerically less decline from baseline at Week 26 with rivastigmine versus placebo on the memory (effect size: 0.26$)$ language (0.27) and praxis (0.24) subscales of the SIB.

\section{Efficacy of rivastigmine on the newly defined SIB subscales: visual; language; working memory/memory; praxis and social skills; and naming}

A significant reduction in decline was seen from baseline at Week 26 in rivastigmine-treated patients compared with placebo on the working memory/memory subscale (Factor 3; $P=0.018$; Figure 1). Despite an apparent trend towards reduced decline in rivastigmine-treated patients compared with placebo on the visual (Factor 1), language (Factor 2), praxis and social skills (Factor 4), and naming (Factor 5) subscales, these differences did not reach significance $(P=0.056, P=0.056, P=0.117$ and $P=0.072$, respectively; Figure 1). Based on effect sizes, numerically less decline was observed in patients randomized to rivastigmine compared with placebo on all five newly defined SIB subscales (visual, 0.21; language, 0.22; working memory/memory, 0.28 ; praxis and social skills 0.12; naming, 0.19). Supporting the ITT-LOCF analysis, the ITT-OC analysis also demonstrated significantly less decline with oral rivastigmine versus placebo on the working memory/memory subscale $(P=0.038)$.

Efficacy of rivastigmine on the individual items of the SIB Calculation of treatment effect sizes for each of the individual items demonstrated efficacy of rivastigmine 
Table 2 Factor scores derived by factor analysis to allocate the SIB items into new subscales

\begin{tabular}{|c|c|c|c|c|c|}
\hline SIB item & $\begin{array}{l}\text { Factor 1: } \\
\text { visual }\end{array}$ & $\begin{array}{l}\text { Factor 2: } \\
\text { language }\end{array}$ & $\begin{array}{l}\text { Factor 3: } \\
\text { working } \\
\text { memory/ } \\
\text { memory }\end{array}$ & $\begin{array}{l}\text { Factor 4: } \\
\text { praxis } \\
\text { and } \\
\text { social skills }\end{array}$ & $\begin{array}{l}\text { Factor } 5 \\
\text { naming }\end{array}$ \\
\hline 1a: Shake hands & 0.18637 & 0.10742 & 0.14187 & 0.46777 & 0.06278 \\
\hline 1b: Follow directions & 0.03926 & 0.16075 & 0.09979 & 0.72810 & -0.02260 \\
\hline 1c: Sit/move to table/pull tray & 0.14512 & 0.07164 & 0.03912 & 0.81773 & 0.09756 \\
\hline 2: Examiner's name (immediate recall) & 0.19391 & 0.01890 & 0.35278 & 0.20451 & 0.00918 \\
\hline 3: Subject's name & 0.13280 & 0.09591 & 0.11500 & 0.27894 & 0.13269 \\
\hline 4a: Write name & 0.14911 & 0.83191 & 0.21540 & -0.01825 & -0.13753 \\
\hline 4b: Copy name & 0.18811 & 0.79331 & 0.22121 & 0.02954 & -0.17792 \\
\hline 5: Current month & 0.09048 & 0.10255 & 0.16135 & -0.00452 & 0.08214 \\
\hline 6: Months of the year & -0.08840 & 0.44571 & 0.16330 & 0.06817 & 0.13195 \\
\hline 7: City & 0.03403 & 0.23398 & 0.47705 & 0.03550 & 0.13178 \\
\hline $8 a+8 b$ : Descriptive naming (cup + spoon) & 0.19170 & 0.11481 & 0.45178 & 0.17732 & 0.53765 \\
\hline 9a: Reading comprehension & 0.04419 & 0.56384 & 0.01299 & 0.09907 & 0.24333 \\
\hline 9b: Verbal comprehension & 0.10071 & 0.58147 & 0.04924 & 0.17725 & 0.22978 \\
\hline 9c: Reading & 0.06602 & 0.75731 & -0.03219 & 0.10638 & 0.12338 \\
\hline 10: Sentence recall & 0.12520 & 0.43543 & 0.27214 & 0.11551 & 0.15668 \\
\hline 11a + 11b: Repetition (people spend money + baby) & 0.17676 & 0.15277 & 0.29899 & 0.46208 & 0.21274 \\
\hline 12: Digit span (series) & -0.01152 & 0.12864 & 0.39120 & 0.09214 & 0.02801 \\
\hline 13: Verbal fluency & 0.22551 & 0.00574 & 0.60501 & 0.16787 & 0.25116 \\
\hline 14: Examiner's name (delayed recall) & 0.02598 & 0.04244 & 0.22845 & 0.06847 & -0.00027 \\
\hline $15+20$ : Naming object in photograph (cup + spoon) & 0.20978 & 0.21774 & 0.18377 & 0.15042 & 0.67844 \\
\hline $16+18:$ Using cup (photograph + object) & 0.24127 & 0.04208 & 0.48144 & 0.06123 & 0.41299 \\
\hline $17+22:$ Naming object (cup + spoon) & 0.19141 & 0.15694 & 0.16444 & 0.19876 & 0.70735 \\
\hline $19+24:$ Forced choice naming (cup + spoon) & 0.34707 & 0.04743 & 0.04278 & 0.33749 & 0.37978 \\
\hline $21+23:$ Using spoon (photograph + object) & 0.24747 & 0.06307 & 0.40561 & 0.15432 & 0.39211 \\
\hline 25: Immediate recall of objects (cup + spoon) & 0.30110 & 0.10494 & 0.46109 & 0.22645 & 0.17432 \\
\hline $26+30 a+30 b$ : Color naming (blue + red + green) & 0.52207 & 0.21932 & 0.07852 & 0.03810 & 0.36658 \\
\hline 27: Color matching & 0.59979 & 0.05950 & 0.12284 & 0.12189 & 0.17387 \\
\hline 28: Colored block & 0.56639 & 0.10328 & 0.02037 & 0.12734 & 0.05381 \\
\hline 29: Color discrimination & 0.68207 & 0.13255 & 0.28264 & 0.15338 & 0.11077 \\
\hline 31: Shape matching & 0.54832 & 0.13483 & 0.23207 & 0.22148 & 0.32796 \\
\hline 32: Shape & 0.55816 & -0.04496 & 0.22437 & 0.19948 & 0.09445 \\
\hline 33: Shape discrimination & 0.58847 & 0.06018 & 0.38125 & 0.26172 & 0.10459 \\
\hline $30 c+34 a+34 b$ : Shape identification (square + circle + triangle) & 0.36032 & 0.26761 & 0.33625 & 0.21808 & 0.20124 \\
\hline 35a: Drawing (circle) & 0.23055 & 0.20295 & 0.28592 & 0.04922 & 0.25047 \\
\hline 35b: Drawing (square) & 0.23820 & 0.30433 & 0.47077 & 0.06087 & 0.13839 \\
\hline 36: Auditory span & 0.21216 & 0.12792 & 0.28611 & 0.27011 & 0.10342 \\
\hline 37: Visual span & 0.26459 & 0.28920 & 0.30645 & 0.17403 & 0.30167 \\
\hline 38: Delayed recall of objects (cup + spoon) & 0.37135 & -0.04291 & 0.47166 & 0.16280 & 0.07484 \\
\hline 39: Orientation to name & 0.15592 & 0.01837 & 0.16938 & 0.58642 & 0.14839 \\
\hline 40: Language ability (free conversation) & 0.16147 & -0.07097 & 0.40767 & 0.47483 & 0.16766 \\
\hline
\end{tabular}

SIB, Severe Impairment Battery. Scores were derived using initial common factor extraction and the principal component method, and were based on data from 217 patients. Estimates of loadings were obtained using varimax rotation. SIB items were allocated to factors on the basis of highest score. Items allocated to each factor are shown in bold. 
Table 3 Allocation of the individual items of the SIB into five new subscales

\begin{tabular}{|c|c|c|}
\hline New subscale & SIB item & SIB domain [10] \\
\hline \multirow[t]{8}{*}{ Factor 1: visual } & $26+30 a+30 b$ : Color naming (blue + red + green) & Language \\
\hline & 27: Color matching & Visuospatial perception \\
\hline & 28: Colored block & Memory \\
\hline & 29: Color discrimination & Visuospatial perception \\
\hline & $30 c+34 a+34 b:$ Shape identification (square + circle + triangle) & Language \\
\hline & 31: Shape matching & Visuospatial perception \\
\hline & 32: Shape & Memory \\
\hline & 33: Shape discrimination & Visuospatial perception \\
\hline \multirow[t]{7}{*}{ Factor 2: language } & 4a: Write name & Language \\
\hline & 4b: Copy name & Language \\
\hline & 6: Months of the year & Language \\
\hline & 9a: Reading comprehension & Language \\
\hline & 9b: Verbal comprehension & Language \\
\hline & 9c: Reading & Language \\
\hline & 10: Sentence recall & Memory \\
\hline \multirow[t]{14}{*}{ Factor 3: working memory/memory } & 2: Examiner's name (immediate recall) & Memory \\
\hline & 5: Current month & Orientation \\
\hline & 7: City & Orientation \\
\hline & 12: Digit span (series) & Attention \\
\hline & 13: Verbal fluency & Language \\
\hline & 14: Examiner's name (delayed recall) & Memory \\
\hline & $16+18:$ Using cup (photograph + object) & Praxis \\
\hline & $21+23$ : Using spoon (photograph + object) & Praxis \\
\hline & 25: Immediate recall of objects (cup + spoon) & Memory \\
\hline & 35a: Drawing (circle) & Construction \\
\hline & 35b: Drawing (square) & Construction \\
\hline & 36: Auditory span & Attention \\
\hline & 37: Visual span & Attention \\
\hline & 38: Delayed recall of objects (cup + spoon) & Memory \\
\hline \multirow[t]{7}{*}{ Factor 4: praxis and social skills } & 1a: Shake hands & Social skills \\
\hline & 1b: Follow directions & Social skills \\
\hline & 1c: Sit/move to table/pull tray & Social skills \\
\hline & 3: Subject's name & Orientation \\
\hline & $11 a+11 b:$ Repetition (people spend money + baby) & Language \\
\hline & 39: Orientation to name & Orientating head to name \\
\hline & 40: Language ability (free conversation) & Language \\
\hline \multirow[t]{4}{*}{ Factor 5: naming } & 8a + 8b: Descriptive naming (cup + spoon) & Language \\
\hline & $15+20$ : Naming object in photograph (cup + spoon) & Language \\
\hline & $17+22:$ Naming object (cup + spoon) & Language \\
\hline & 19 + 24: Forced-choice naming (cup + spoon) & Language \\
\hline
\end{tabular}

SIB, Severe Impairment Battery. SIB domain allocation refers to the nine domains of the SIB, to which each of the items have been previously assigned. New subscales relate to the new factors generated from factor analysis of the 40 individual SIB items. 


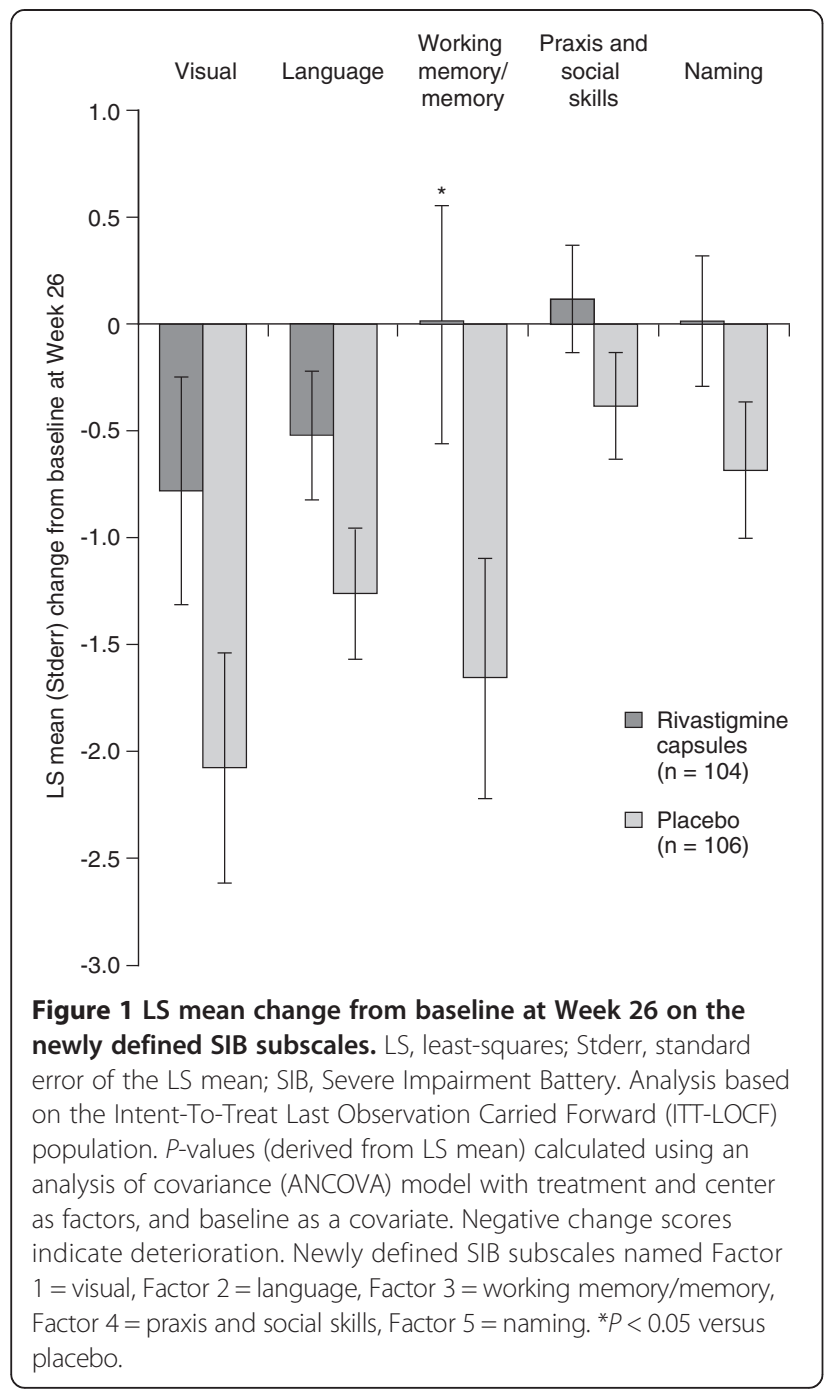

on a broad range of SIB items, across each of the newly defined SIB subscales (Figure 2). Greatest effect sizes ( 0.30 and above) were observed on items assessing the current month (item 5; effect size 0.30) and digit span series (item 12; 0.33).

Effect sizes above 0.2 (range 0.21 to 0.28 ) were observed on these items: shake hands (1a), write name (4a), copy name (4b), city (7), verbal comprehension (9b), naming object (cup + spoon; 17+22), using spoon $(21+23)$ and shape discrimination (33). Effect sizes of 0.1 and above (range 0.10 to 0.19 ) were observed on the following items: directions (1b), subject's name (3), reading comprehension (9a), naming object in photograph $(15+20)$, using cup $(16+18)$, color naming $(26+30 a+30 b)$, color block (28), shape identification $(30 c+34 a+34 b)$, drawing circle (35a), auditory span (36), and orientation to name (39). For all other items (1c, 2, 6, 8a +8b, 9c, 10, 11a +11b, 13, 14, 19+24, 25, $27,29,31,32,35 \mathrm{~b}, 37,38$ and 40 ) effect sizes were $<0.1$, and considered similar between rivastigmine and placebo groups, with the exception of item 25 (immediate recall) for which the calculated effect size of -0.18 suggested better performance in the placebo group.

\section{Discussion}

$\mathrm{AD}$ is associated with gradual loss of cholinergic transmission in the brain, which manifests as progressive deterioration of cognitive function [14]. As cholinergic deficits correlate with disease stage, treatment strategies that increase levels of acetylcholine available in cholinergic synapses may become more relevant as the disease progresses.

Several clinical studies and retrospective analyses of trial databases have investigated the efficacy of rivastigmine in patients with moderately severe AD. Analysis of a 52 -week study of oral rivastigmine investigated the long-term efficacy of rivastigmine in patients with $\mathrm{AD}$ stratified by baseline dementia severity, determined using the GDS [15]. Patients with a GDS score $\geq 5$ (moderately severe disease) receiving 1 to $4 \mathrm{mg} /$ day or 6 to $12 \mathrm{mg} /$ day rivastigmine capsules showed significant improvements compared with placebo on the ADAS-cog during an initial 26-week, double-blind treatment phase [15]. At Week 52, following a further 26 weeks of open-label treatment with 6 to $12 \mathrm{mg} /$ day rivastigmine, ADAS-cog performance was significantly superior in treated patients compared with projected placebo [15]. These findings suggest that rivastigmine may have sustained cognitive benefits in patients with moderately severe AD. Furthermore, retrospective, pooled analysis of data from three randomized, placebocontrolled, double-blind, six-month clinical trials of patients with probable AD (MMSE score of 10 to 26) provided evidence for a cognitive benefit of rivastigmine treatment in the subset of patients with more severe AD (MMSE score of 10 to 12) [7]. In support of these findings, further pooled analysis of these studies, in which patients randomized to receive 6 to $12 \mathrm{mg} /$ day rivastigmine capsules were stratified according to dementia severity, reported significant efficacy of rivastigmine versus placebo on the ADAS-cog across all disease stages, including moderately severe AD (MMSE score of $\leq 15)$ [16].

Although these analyses suggest cognitive benefits of rivastigmine in moderately severe $\mathrm{AD}$, the scales commonly used to assess cognitive efficacy in clinical trials of patients with mild-to-moderately severe $\mathrm{AD}$, for example, the ADAS-cog, may not be sensitive to the full range of cognitive changes in patients with severe $A D$, owing to floor effects and limited ability of these patients to complete assessments. Tests designed to be sensitive within the capabilities of patients with more advanced stage $A D$, such as the $\mathrm{SIB}$, may provide further insight 


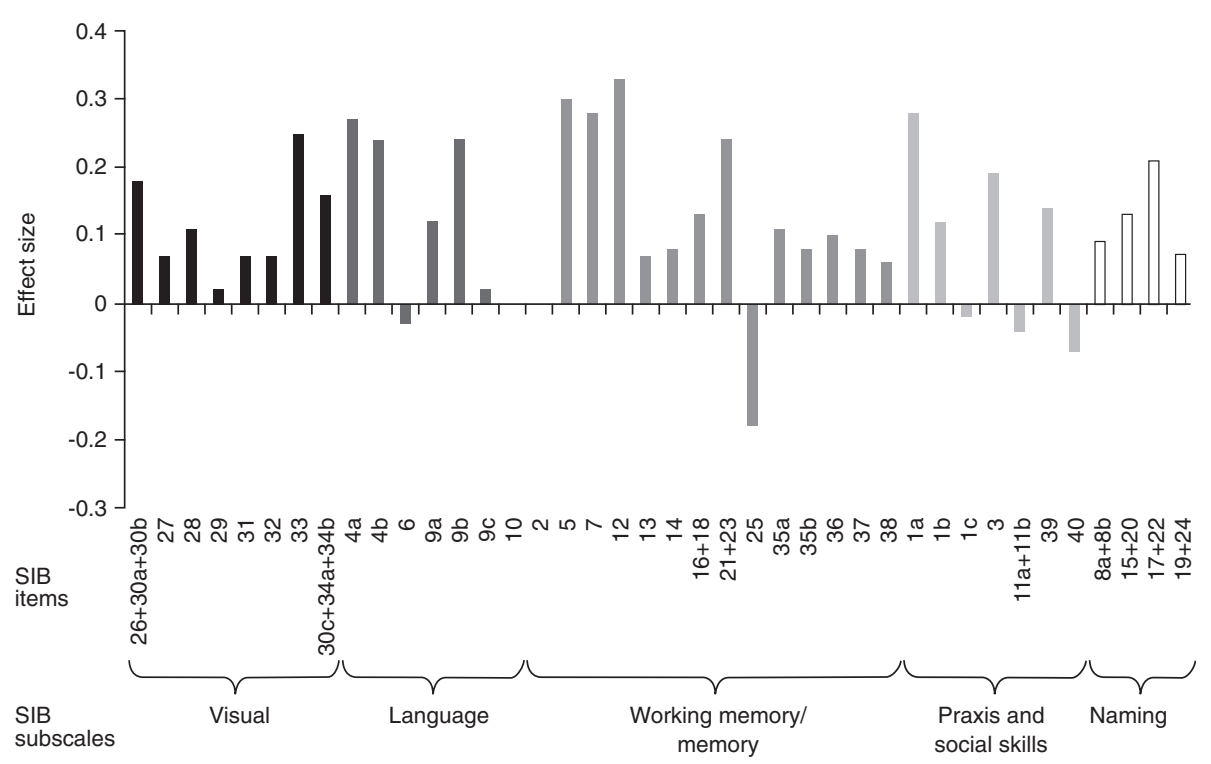

Figure 2 Effect sizes based on mean change from baseline at Week 26 on individual SIB items. SIB, Severe Impairment Battery. Analysis based on the Intent-To-Treat Last Observation Carried Forward (ITT-LOCF) population. Negative change scores indicate deterioration.

into the efficacy of rivastigmine on specific aspects of cognitive function.

A previous randomized, placebo-controlled clinical trial reported significant efficacy of rivastigmine on the total SIB in patients with moderately severe-to-severe AD [9]; SIB data from this trial formed the basis of the current analysis, in which we sought to further investigate the pattern of cognitive efficacy of rivastigmine in patients with severe AD. Rivastigmine treatment was associated with significant efficacy on the higher-order memory and language subscales of the SIB, as defined by a previous analysis of the nine SIB domains [13]. A trend toward numerically greater efficacy was also observed on the praxis subscale.

In the version of the SIB used in this analysis, the nine domains of the SIB comprised 40 items, which assess a diverse range of cognitive impairments, from awareness of the current month, to ability to identify everyday objects, for example, a cup and spoon. Given the broad range of cognitive functions assessed by the items encompassed in the nine SIB domains, we performed a new factor analysis of the SIB using the individual items, as opposed to domains, which allocated the 40 items to one of five factors (two more than the previously published analysis). Rivastigmine demonstrated significant efficacy compared with placebo on the new subscale we termed working memory/memory, based on the range of SIB items encompassed in this subscale. Based on effect sizes, a numerical trend toward greater efficacy was also observed on each of the newly defined subscales in rivastigmine-treated patients compared with placebo. The lack of a significant treatment effect on the visual, language, praxis and social skills, and naming factors may have resulted from floor and ceiling effects on some individual SIB items. Since the majority of studies in AD have been performed in patients of mild-to-moderate disease stage, issues related to these effects in patients with severe $A D$ have not been routinely addressed in clinical studies. In the current analysis, it is possible that floor or ceiling effects at baseline may have influenced loading on to factors, and the ability to observe treatment effects versus placebo.

In addition to investigating efficacy on previously defined and newly defined subscales, we also calculated the magnitude of treatment effects on individual SIB items. Efficacy on these individual items may translate into clinically meaningful benefits for patients and their caregivers. Greatest effect sizes were observed on items assessing current month and memory span (digit series). Both of these items form part of the newly defined working memory/memory subscale and the previously defined memory subscale, and may account in part for the significant efficacy of treatment observed on these subscales. However, overall, rivastigmine was shown to demonstrate efficacy on a wide range of items (based on effect sizes), spanning across both the newly defined and previously defined SIB subscales. These findings suggest that the observed efficacy of rivastigmine on the SIB is not a result of efficacy on a few individual items or a few cognitive domains, but rather is a fairly consistent, 
cumulative effect across a wide range of cognitive tasks. These findings are encouraging, as they suggest rivastigmine may have broad cognitive efficacy in severe AD.

Limitations of this study include its retrospective nature; this was an exploratory analysis and was only intended to be hypothesis forming. This study was not powered to detect differences on the individual SIB items, previously defined subscales, or on the new subscales derived by factor analysis of the SIB items. These considerations may reduce the robustness of these results. Larger studies are needed to confirm these findings, and to further investigate the cognitive efficacy of oral rivastigmine in patients with severe AD.

Additional information on the pattern of cognitive benefit of rivastigmine in patients with severe $\mathrm{AD}$ is provided by the ACTION study (ClinicalTrials.gov identifier NCT00948766) [8]. The SIB was a co-primary outcome measure to assess cognition in this study. The ACTION study demonstrated significantly greater efficacy of $13.3 \mathrm{mg} / 24 \mathrm{~h}$ versus $4.6 \mathrm{mg} / 24 \mathrm{~h}$ rivastigmine patch on cognition, as measured by the change from baseline at Week 24 on the total SIB score, without a marked impact on safety and tolerability [8]. To our knowledge, this was the first clinical trial of the rivastigmine patch in patients with severe AD; primary and post-hoc analyses of data obtained in this study will allow investigation into the use and specific cognitive effects of the high dose $13.3 \mathrm{mg} / 24 \mathrm{~h}$ rivastigmine patch in this indication. Furthermore, given that $13.3 \mathrm{mg} / 24 \mathrm{~h}$ patch is now approved for the symptomatic treatment of severe AD in the USA [5], investigating physician experiences with high-dose patch in this indication will provide further information on how trial findings may translate into clinical practice.

\section{Conclusions}

In this post-hoc analysis, oral rivastigmine demonstrated efficacy on the previously defined memory and language subscales, newly defined working memory/memory subscales, and several individual items of the SIB in patients with moderately severe-to-severe AD. The cumulative effect observed across a broad range of tasks corroborates previous evidence, and reinforces that rivastigmine may be an effective therapy in the treatment of severe AD.

\footnotetext{
Abbreviations

ACTION: ACTivities of daily living and cognit/ON; AD: Alzheimer's disease; ADAS-cog: Alzheimer's Disease Assessment Scale-cognitive subscale: ADCS-ADL: Alzheimer's Disease Cooperative Study-Activities of Daily Living; ADCS-CGIC: Alzheimer's Disease Cooperative Study-Clinical Global Impression of Change; ADL: Activities of daily living; ANCOVA: Analysis of covariance; GDS: Global Deterioration Scale; ITT-LOCF: Intent-To-Treat Last Observation Carried Forward; ITT-OC: Intent-To-Treat Observed Case; LS: Least-squares; MMSE: Mini-Mental State Examination; NPI-10: 10-item Neuropsychiatric
}

Inventory; NPI-4: 4-item Neuropsychiatric Inventory; SD: Standard deviation; SIB: Severe Impairment Battery; Stderr: Standard error of the LS mean.

\section{Competing interests}

SF has served as a scientific consultant to companies marketing, developing or contributing to the development of treatments for cognition, including Accera, Baxter, Bristol-Myers Squibb, Cebria, Dart Neuroscience, Eisai, Janssen Al, Eli Lilly, Lundbeck, Lupin, MedAvante, Merck, Neuronix, Neurotrack, Targacept and United Biosource. There was no payment from Novartis for participation in this article. His institution has received grant/contract support for clinical trials from Accera, Dart Neuroscience, Eisai, Eli Lilly, EnVivo, GE Healthcare, Genentech, Janssen, Janssen Al, Lundbeck, Merck, Roche, Neuronix, Takeda and Bristol-Myers Squibb. He also has stock options from Accera, Intellect Neurosciences and MedAvante, and stock in Lexicon Pharmaceuticals. SK has no relevant conflicts of interest to declare. MS and XM are full-time employees and stock holders of Novartis Pharmaceuticals Corporation, East Hanover, NJ, USA. Novartis developed and manufactures rivastigmine, and sponsored the large, multicenter, randomized, double-blind trials that led to the approval of rivastigmine in the USA for mild-tomoderate and severe AD. Novartis also sponsored the study of oral rivastigmine in moderately severe-to-severe AD which forms the basis of the current analysis.

\section{Authors' contributions}

SF, SK, MS and XM made substantial contributions to the analysis and interpretation of the data, were involved in drafting the manuscript or revising it critically for important intellectual content and have given final approval of the version to be published.

\section{Acknowledgments}

The current data analyses were supported by Novartis Pharmaceuticals Corporation, East Hanover, NJ, USA. Katy Cooke of Fishawack Communications Ltd, Oxford, UK, provided medical writing and editorial support in the production of this manuscript; this service was sponsored by Novartis Pharmaceuticals Corporation, East Hanover, NJ, USA. The article-processing charge was sponsored by Novartis Pharmaceuticals Corporation, East Hanover, NJ, USA.

\section{Author details}

${ }^{1}$ Alzheimer's Disease Center, Comprehensive Center on Brain Aging, NYU Langone Medical Center, Room 506, 145 East 32nd Street, New York, NY 10016, USA. ${ }^{2}$ Novartis Pharmaceuticals Corporation, East Hanover, NJ 07936-1080, USA.

Received: 1 November 2013 Accepted: 6 December 2013 Published: 18 December 2013

References

1. Schneider LS, Anand R, Farlow MR: Systematic review of the efficacy of rivastigmine for patients with Alzheimer's disease. Int J Geriatr Psychopharmacol 1998, 1:S26-S34.

2. Corey-Bloom J, Anand $\mathrm{R}$, Veach J: A randomized trial evaluating the efficacy and safety of ENA 713 (rivastigmine tartrate), a new acetylcholinesterase inhibitor, in patients with mild to moderately severe Alzheimer's disease. Int J Geriatr Psychopharmacol 1998, 1:55-65.

3. Rösler M, Anand R, Cicin-Sain A, Gauthier S, Agid Y, Dal-Bianco P, Stähelin $H B$, Hartman R, Gharabawi M: Efficacy and safety of rivastigmine in patients with Alzheimer's disease: international randomised controlled trial. BMJ 1999, 318:633-638.

4. Finkel SI: Effects of rivastigmine on behavioral and psychological symptoms of dementia in Alzheimer's disease. Clin Ther 2004, 26:980-990.

5. Exelon Patch ${ }^{\oplus}$ US prescribing information, [http://www.pharma.us.novartis. com/product/pi/pdf/exelonpatch.pdf]

6. Exelon ${ }^{\oplus}$ US prescribing information, [http://www.pharma.us.novartis.com/ product/pi/pdf/exelon.pdf

7. Burns A, Spiegel R, Quarg P: Efficacy of rivastigmine in subjects with moderately severe Alzheimer's disease. Int J Geriatr Psychiatry 2004 19:243-249.

8. Farlow MR, Grossberg GT, Sadowsky CH, Meng X, Somogyi M: A 24-week, randomized, controlled trial of rivastigmine patch $13.3 \mathrm{mg} / 24 \mathrm{~h}$ versus 
$4.6 \mathrm{mg} / 24 \mathrm{~h}$ in severe Alzheimer's dementia. CNS Neurosci Ther 2013, 19:745-752.

9. Lopez-Pousa S, Vilalta-Franch J, Hernandez B, Rapatz G: Efficacy of rivastigmine in patients with severe Alzheimer's disease: a double-blind, randomized pilot study. Brain Aging 2004, 4:26-34.

10. Panisset M, Roudier M, Saxton J, Boller F: Severe impairment battery. A neuropsychological test for severely demented patients. Arch Neurol 1994, 51:41-45.

11. Schmitt FA, Ashford W, Ernesto C, Saxton J, Schneider LS, Clark CM, Ferris SH, Mackell JA, Schafer K, Thal LJ: The severe impairment battery: concurrent validity and the assessment of longitudinal change in Alzheimer's disease. The Alzheimer's Disease Cooperative Study. Alzheimer Dis Assoc Disord 1997, 11:S51-S56.

12. Saxton J, McGonigle-Gibson K, Swihart A, Miller M, Boller F: Assessment of the severely impaired patient: description and validation of a new neuropsychological test battery. Psychol Assess 1990, 2:298-303.

13. Schmitt FA, van Dyck CH, Wichems CH, Olin JT, Memantine MEM-MD-02 Study Group: Cognitive response to memantine in moderate to severe Alzheimer disease patients already receiving donepezil: an exploratory reanalysis. Alzheimer Dis Assoc Disord 2006, 20:255-262.

14. Bartus RT, Dean RL 3rd, Beer B, Lippa AS: The cholinergic hypothesis of geriatric memory dysfunction. Science 1982, 217:408-414.

15. Doraiswamy PM, Krishnan KR, Anand R, Sohn H, Danyluk J, Hartman RD, Veach J: Long-term effects of rivastigmine in moderately severe Alzheimer's disease: does early initiation of therapy offer sustained benefits? Prog Neuropsychopharmacol Biol Psychiatry 2002, 26:705-712

16. Kurz A, Farlow M, Quarg P, Spiegel R: Disease stage in Alzheimer disease and treatment effects of rivastigmine. Alzheimer Dis Assoc Disord 2004, 18:123-128.

doi:10.1186/alzrt229

Cite this article as: Ferris et al:: Rivastigmine in moderately severe-tosevere Alzheimer's disease: Severe Impairment Battery factor analysis. Alzheimer's Research \& Therapy 2013 5:63.

\section{Submit your next manuscript to BioMed Central and take full advantage of:}

- Convenient online submission

- Thorough peer review

- No space constraints or color figure charges

- Immediate publication on acceptance

- Inclusion in PubMed, CAS, Scopus and Google Scholar

- Research which is freely available for redistribution 